\title{
Retórica digital rizomática: percepción de formación digital de universitarios a diez años de su experiencia con Enciclomedia.
}

\section{Rhizomatic digital rhetoric: perception of digital training of university students ten years after their experience with Enciclomedia.}

\author{
Ismene Ithaí Bras Ruiz \\ Universidad Nacional Autónoma de México \\ ismene_bras@cuaed.unam.mx
}

\begin{abstract}
Resumen
El texto presenta los resultados de la investigación en la que se valoró percepción en la formación digital en estudiantes universitarios a partir de su experiencia con el programa Enciclomedia en México hace diez años, hasta llegar a la universidad. Se parte del supuesto de que esta formación no se limitó a este programa, sino que se experimentó en varios entornos con el mundo digital, aunque Enciclomedia fue la primera de tipo académico en sus vidas. Los resultados muestran que aunque la percepción de este programa no es del todo positiva, se considera que estas prácticas digitales tuvieron un impacto en la formación digital a la larga, al igual que la experimentación con el celular de los padres, videojuegos, videos, navegación. Estas prácticas son denominadas rizomáticas, en virtud de son entramados interconectados y son una forma de retórica digital en tanto que nos muestra la evolución de la narrativa subjetiva y compleja de los estudiantes.
\end{abstract}

Palabras clave: Retórica digital, Rizoma, Formación digital, Enciclomedia, Universitarios

\begin{abstract}
The text presents the results of the research in which perception was assessed in digital training in university students from their experience with the Enciclomedia program in Mexico ten years ago, until they arrived at the university. It is based on the assumption that this training was not limited to this program but was experienced in various environments with the digital world, although Enciclomedia was the first academic type in their lives. The results show that although the perception of this program is not entirely positive, it is considered that these digital practices had an impact on the digital training in the long run, as well as the experimentation with the parents' cell phone, video games, videos, navigation. These practices are called rhizomatic, because they are interconnected frameworks and are a form of digital rhetoric while showing the evolution of the subjective and complex narrative of the students.
\end{abstract}

Key words: Digital rhetoric, Rhizome, Digital training, Encyclomedia, University students

\section{Introducción}

La integración de la tecnología educativa ha pasado por diversos tipos de evaluaciones que van desde aspectos de administración, gestión, presupuestal, hasta de rendimiento, pedagógicos como cognitivos, entre otros. Dentro de las dinámicas que han introducido la importancia de las nuevas tecnologías en los proyectos educativos, se sitúa en las distintas conformación, interpretaciones y lecturas del mundo digital (retóricas digitales) y las prácticas en estos escenarios. La relación entre estos 
aspectos y la formación de los alumnos atraviesa a su vez, por la narrativa de distintos agentes que van desde los políticos, los administradores de los proyectos de integración de tecnología educativa, los directores de las escuelas, madres y padres de familia, pero también amigos, y la de los propios estudiantes.

La retórica digital que se busca recuperar, se centra pues en la recuperación de estos entramados y narrativas en tanto que construcción de prácticas en el mundo digital, que han ido formando a los alumnos desde la educación básica hasta la universidad. Valorar la integración de las Tecnologías de la Información y la Comunicación (TIC), desde la retórica digital coadyuva a los reportes e informes, para entender cómo a la larga esa práctica se transforma en hábito y después en competencia. Si bien el objetivo de este texto no se sitúa en estos dos últimos aspectos, sino en la evolución de esas prácticas y hacer visibles los distintos entornos de formación digital, es importante destacar que una práctica que lleva más de 10 años modifica la lectura del mundo.

El estudio que se presenta tiene como marco el proyecto sobre cultura y retórica digital en las comunidades universitarias, en el que se valora desde un enfoque complejo de la realidad, la importancia de la formación digital desde varios entornos, y no de carácter lineal. Por ello, se presume que esta formación digital no ha provenido únicamente de los programas políticos educativos, sino que se conforma de un entramado de experiencias académicas, sociales, familiares y subjetivas de tipo rizomático. Es decir, que las prácticas educativas que integran las TIC son una parte del todo formativo y que evolucionan en la medida que los estudiantes se las apropian y las socializan en distintos ámbitos, incluido el educativo.

El estudio se justifica en virtud de que la mayoría de quienes se encuentran en estos momentos en las aulas universitarias en el país, en su niñez aún alcanzaron a tener la experiencia del programa Enciclomedia en el período 2009-2010. Si bien éste concluyó entre 2010 y 2011, habría que sumar que para los siguientes años aumentó el número de programas de integración de las TIC en diversos contextos ya fuera a través de programas específicos a nivel de los estados o de las entidades de formación del nivel bachillerato. No se busca analizar y valorar el programa Enciclomedia por sí mismo, sino como entorno inicial del estudio 
que se presenta, esto es interacciones iniciales con la tecnología educativa integrada desde la óptica del gobierno mexicano en los estudiantes más jóvenes.

La figura 1 muestra justamente como las retóricas digitales entre instituciones y las subjetividades de los agentes pueden ser diferentes en su concepción y en su desarrollo.
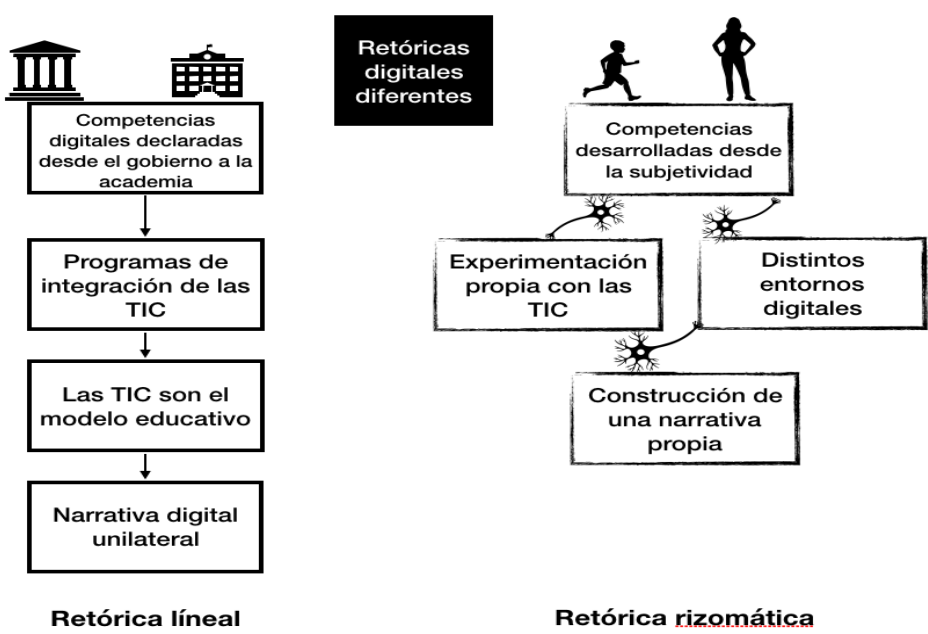

Figura 1. Retórica digital lineal vs retórica digital rizomática Fuente: Autoría propia

\section{La retórica digital como estudio de las prácticas digitales}

La retórica digital permite la articulación de varias narrativas sociales entre diversos agentes de manera no estructurada que implica condiciones materiales y metamateriales de la praxis digital. Ésta a su vez, se relaciona con la cultura digital en tanto que, como planteara Levy (2007) hace más de diez años:
[...] abarca más allá de los sistemas, prácticas, entornos y medios culturales simbólicos (como los directamente relacionados con la información, la comunicación, el conocimiento o la educación) y se extiende prácticamente por todos los ámbitos de la sociedad digital, los rápidos procesos de innovación desencadenados por las TIC digitales han transformado radicalmente, junto con los sistemas, los colectivos y las dinámicas de la información y la comunicación, también las formas de conocimiento e investigación tecno-científica (Lèvy, 2007, p. 129).

Así que la retórica digital se opone a la tradicional en tanto que analiza las prácticas y las construcciones cognitivas de manera rizomática. Se trata de un área de estudio que en realidad tiene más de 30 años y que a decir de Zallo (2011) pasa "Del discurso racional, vertical y prescriptivo 
de la alta cultura se pasa a la cultura que el usuario construye fragmentando y mezclando el orden abstracto alfanumérico de la lectoescritura con el orden más sensitivo de imágenes y con el conocimiento intuitivo, de prueba y error, dando lugar a nuevas significaciones (Zallo, 2011, p.104)". De ahí que estas significaciones creen espacios de estudio que crean narrativas propias, subjetivas y entrelazadas para agentes que cada vez interconectados. Los distintos entornos en los que nos movemos nos permiten formarnos una retórica en la no sólo simbolizamos el mundo sino todos sus procesos mediante actividades, sino que literalmente lo nombramos y conocemos mediante distintos procesos, dispositivos y declaraciones que damos. Se trata entonces de una adopción y adaptación de una narrativa, en tanto que prácticas e interacciones, que: “[La] retórica digital no es meramente la suma de la tecnología a la retórica -o viceversa- y revolverlas. El concepto de retórica digital requiere atención sustancial a las formas en las que la retórica cambia en una era tecnológica y cómo la tecnología es formada por la expresión humana, tanto sobre cómo y a través de la propia tecnología" (Hess, 2017, p.2).

En su texto Digital Rhetoric: Theory, Practice, and Property, Lanham (1992) advertía la necesidad de reflexionar sobre los aspectos sociales tanto como en los epistémicos y gnoseológicos en torno a la dinámica de los humanos con las computadoras, no por otra cosa sino porque lo que la computadora necesitaba ser explicada en términos de la realización del pensamiento humano. Y para 1993 integra estas reflexiones al quehacer de los universitarios a partir del hipertexto en The Electronic Word: Democracy, Technology, and the Arts. Autores como Moulthrop (1994), Landow (1994) o Bolter (2001) habían comenzado a establecer que el vínculo con el mundo digital en especial de las computadoras estaba creando interacciones particulares a partir del hipertexto, las formas literarias pero también del conocimiento rizomático. Estos aspectos se estarían ampliando con la venta masiva de laptops, abaratamiento de la conexión a Internet, la aparición de los teléfonos inteligentes, las redes sociales digitales, el aumento de recursos y contenidos en la Web, y la consolidación de la Web 2.0.

En este contexto, los estudios sobre retórica digital ampliaron aún más su campo de estudio desde principios de siglo, poniendo interés en la construcción de narrativas desde la visión de las esferas políticas del uso 
de la tecnología (Losh, 2009), hasta los aspectos comunicativos y sociales del uso en red de la tecnología a partir de la ubicuidad (Pfister, 2014), hasta llegar al análisis de nuevas dinámicas sociales que se generan por el uso de las TIC (Eyman, 2015). En consecuencia en palabras de Zappen:

Una teoría de la retórica digital debe reconocer cómo la retórica tradicional de la persuasión se ha transformando en espacios digitales, invita a tales preguntas $y$, por lo tanto, ofrece nuevas oportunidades de investigación en teoría y crítica de la retórica y una visión ampliada de lo que la retórica de la ciencia y la tecnología podría convertirse dentro de la teoría, próxima década y más allá (Zappen, 2005, p. 324).

De lo anterior se desprende que los estudios sobre retórica digital parten de una reconstrucción de las prácticas socio-digitales que es lo que forma la cultura, en la que los distintos agentes de manera rizomática van interconectando su visión de la tecnología hasta formar un entramado sumamente complejo, pero que en cada caso nos refiere cómo los agentes forman sus culturas digitales.

Si asumimos que la cultura digital involucra a varios agentes y que esta agencia de cada uno justamente busca hacerse de un lugar, podremos entender que el estudio de la retórica nos permite entender porqué hay proyectos o iniciativas, en distintos planos, incluyendo la educación, en los que estos agentes chocan o difieren, pero también abonan de uno y otro modo a la formación digital de cada uno. En este marco, Enciclomedia puede ser entendido como una narrativa digital del estado mexicano de 2003 hasta 2011, en el que se apostó a la integración de las TIC en los espacios de educación básica, bajo el entendido de que se mejoraría el rendimiento escolar. No obstante, además de los diversos contratiempos y problemas que se dieron para implementar este programa, desde el análisis retórico digital, se observó que coexistieron distintas narrativas digitales entre políticos, administradores, profesores, y niños. Siendo estos últimos en quienes debía recaer el beneficio del programa, a la larga fueron haciéndose de su propia narrativa digital no sólo por la experiencia con este programa sino, los caminos rizomáticos que en los que anduvieron. A casi diez años de su finalización, cabe la reflexión sobre los aspectos retóricos digitales en los que se dio el programa y cómo se ven reflejados en la vida universitaria de aquellos niños. 


\section{La retórica digital del proyecto Enciclomedia en México}

Esta iniciativa no fue única en la región, también aparecieron en otros países latinoamericanos como "en Brasil la Educa Red; en Colombia, el programa Computadores para educar; en Ecuador, Edufuturo; [...] Web escuela, en Paraguay" (Elizondo, et al, 2006, p.212). En buena medida lo que rodea a estos proyectos es por una parte la idea de que integrar las TIC a las aulas de la educación básica mejoraría los procesos de enseñanza y aprendizaje. Por el otro, se inscribió una narrativa centrada en que las Sociedades de la Información y el Conocimiento generarían mejoras socio-económicas en la población, y que por lo tanto era menester llevar al alumnado más joven a integrarse a esta dinámica. Este determinismo político-digital implica una imposición de una narrativa propia acerca de los mecanismos didácticos para el aprendizaje y la falta de un modelo educativo que integre las TIC en el universo de prácticas que ya estaban gestando tanto en profesores como en niños en ese momento. Esta retórica pasa por la confusión de que el dispositivo electrónico crea conocimiento o ayuda a tenerlo, pero no a co-construirlo. El enfoque significativo y constructivista desde el Estados, que identifica la tecnología con la innovación, deja de lado el conocimiento rizomático o en red, para plantearse en la práctica sobre una linealidad y transferencia unidireccional así como seguimiento de instrucciones. "El uso de la información que se transmite a través de las TIC requiere un análisis no tan sólo de las características tecnológicas de los dispositivos electrónicos, sino su utilización como herramientas cognitivas o pedagógicas, en vista de su potencialidad para la construcción de conocimientos" (Loredo et al., 2010, p.4).

En el caso de México, Enciclomedia fue un programa que se diseñó para dotar a los salones de 5ㅇ y 6으 de las escuelas primarias mexicanas un pizarrón interactivo, un equipo de computo (manejado por los profesores), acceso a la red, impresora, proyector, el mobiliario para el equipo y el software correspondiente. Su objetivo como tal planteado fue:

Contribuir a la mejora de la calidad de la educación que se imparte en las escuelas públicas de educación primaria del país e impactar en el proceso educativo y de aprendizaje por medio de la experimentación y la interacción de los contenidos educativos incorporados al Programa Enciclomedia, convirtiéndolo en una herramienta de apoyo a la labor docente que estimula nuevas prácticas pedagógicas en el aula para el tratamiento de los temas y contenidos de los Libros de Texto. (Enciclomedia, 2004, p.10) 
Constituído de diez objetivos específicos, se observa principalmente el aspecto de la integración de las TIC en distintos entornos (urbano, rural e indígena) a partir de la idea de que las fuentes de información deben estar actualizadas y contar con recursos tecnológicos novedosos; si bien se apela también al aprendizaje significativo no se aprecia un proyecto educativo de fondo que arropara a Enciclomedia. Existieron otros aspectos como recuperación de conocimientos y experiencias de los docentes, innovación didáctica, organización del conocimiento, obtención de información para valorar el impacto del programa, construcción de redes, trabajo conjunto con la sociedad entre otros (Enciclomedia, 2004, p.1011). Sin embargo, llama la atención especialmente el énfasis en la tecnología como factor de mejora en los procesos de enseñanzaaprendizaje. De su análisis se desprende, como señala Tinajero (2015) que: "Los objetivos descritos y el desarrollo de los materiales sugieren, ante todo, una racionalidad educativa: el acento se ha colocado que dichos recursos tecnológicos ayudarán al mejoramiento de los procesos de enseñanza y aprendizaje" (2015, p. 346)

La base del planteamiento se centra entonces en la convergencia de recursos sobre un espacio de trabajo único, más que una convergencia de medios, que sigue la secuencia instruccional diseñada por el docente. Si bien es cierto que este tipo de iniciativas educativas ponen el énfasis en la necesidad de dotar a los educandos de estrategias más innovadoras, se perdió de vista no sólo el aspecto del modelo educativo que arropara tal integración de las TIC, sino la diversidad y complejidad de los contextos tanto de profesores como de los alumnos. Es así como la incorporación de recursos audiovisuales, visuales y sonoros "no como adorno sino como texto cargado de significación" (Elizondo et al, 2006, p.216), se centra en el uso de canales sensoriales a través de medios digitales, que de manera tradicional no se usan regularmente, pese a que los propios libros de textos tienen actividades que invitan a ello. De ahí que, se asuma que existe un cambio en el paradigma educativo pasivo. El aspecto que preocupa, y sobre este gira el texto presentado, es que se haya asumido que la cultura digital de los alumnos estaría partiendo exclusivamente de las actividades mediadas que los niños a principios de siglo estuvieron realizando al interior de las escuelas y no de un entramado de entornos de prácticas digitales, y que cada uno de éstos fueron completamente diferentes en cada caso. 
En el año 2003 inició sus trabajos el proyecto Enciclomedia y concluyó en 2011. A nivel estrategia se planteó dos líneas de trabajo: la primera se centró en la capacitación de profesores y asesores técnicopedagógicos; la segunda, más cercana al momento de la operación, se trató de canales de acompañamiento tanto en los aspectos pedagógicos como técnicos para el manejo del software (SEP, 2006 p.35). El proceso de equipamiento se dio en el ciclo escolar 2004-2005, para casi 21 mil aulas (Tinajero, 2015). Para 2006 el presupuesto para este proyecto fue de aproximadamente de un equivalente a 53869706 dólares (SEP, 2006) con un total de 165615 aulas de 5 o y 6 o con equipo funcionando en el que trabajaron 3,9 millones de estudiantes de estos grados, mientras que en 2010 se gestionaron 146996 aulas debido a problemas técnicos, robo y de daño de equipo como consecuencia inundaciones por cuestiones metereológicas.

A nivel de contenidos el proyecto de Enciclomedia comprendió los libros de textos gratuitos digitalizados, con ejercicios, simulaciones, audios, videos mapas y visitas virtuales (Barrera, 2015, p.346). Además de esto, tenía un sitio para el trabajo de alumnos y uno más el profesorado con dinámicas para cada uno y acceso a contenidos de la Enciclopedia Encarta de Microsoft, con quien el gobierno mexicano había firmado un convenio, así como paquetes didácticos para el apoyo de estas actividades (ver cuadro 1).

Cuadro 1. Recursos de los sitios que comprendía el proyecto de Enciclomedia Sitio del alumno Sitio del profesor Sitio de Enciclomedia

\begin{tabular}{|c|c|c|}
\hline $\begin{array}{l}\text { Libros de Texto Gratuitos } \\
\text { de } 5 \text { o y } 6 \text { a de educación } \\
\text { básica }\end{array}$ & $\begin{array}{l}\text { Plan y programas de } \\
\text { estudio }\end{array}$ & Actividades interactivas \\
\hline Constitución mexicana & Avance programático & Fonoteca / Biblioteca \\
\hline Atlas de México & $\begin{array}{l}\text { Ficheros de apoyo a los } \\
\text { libros de Español y } \\
\text { Matemáticas }\end{array}$ & $\begin{array}{l}\text { Diagrama temático / } \\
\text { Herramientas }\end{array}$ \\
\hline $\begin{array}{l}\text { Atlas de geografía } \\
\text { universal }\end{array}$ & Sugerencias didácticas & $\begin{array}{l}\text { Microsoft Enciclopedia } \\
\text { Encarta }\end{array}$ \\
\hline Vínculos & $\begin{array}{l}\text { Taller creativo para } \\
\text { apoyar actividades de } \\
\text { música, danza, artes } \\
\text { plásticas y teatro }\end{array}$ & $\begin{array}{l}\text { Filmoteca/ Galería de arte } \\
\text { / Mapas y mapoteca / } \\
\text { Videos/Visitas virtuales }\end{array}$ \\
\hline
\end{tabular}




\begin{tabular}{lll}
\hline Ruleta & Formación docente & $\begin{array}{l}\text { Libros digitalizados y } \\
\text { libros enciclomediados }\end{array}$ \\
\hline \begin{tabular}{l} 
Otras herramientas de $\begin{array}{l}\text { Enciclomedia } \\
\end{array}$ \\
\cline { 2 - 3 }
\end{tabular} & Libros para los profesores & Red Escolar \\
\cline { 2 - 3 } & Curso de inglés & SEPiensa \\
\hline
\end{tabular}

Fuente: Elaborado con información del documento base de Enciclomedia (2003)

Si bien Enciclomedia en buena medida tuvo una retórica basada en el hipertexto, basada en la relación de contenidos de los libros de texto gratuitos con fichas, ejercicios y contenidos de Encarta, no se tenía la intención de relacionarlos con acceso a otros contenidos de la red, en buena medida porque se asumió que había deficiencias en varios espacios de búsqueda de ese momento y que los enlaces entre los textos, fichas y Encarta sería más dinámico para el planteamiento del proyecto. No obstante, la narrativa del hipertexto por el período en el se dio esta iniciativa, sólo respondió al propio espacio de Enciclomedia, es decir que la interacción si bien ya tiene elementos web 2.0, se limita a una plataforma principalmente.

Visto así se puede coincidir con Leal y Arias (2009) cuando señala que fueron los profesores quienes articularon "la mecánica de este discursos, que son quienes presentan y conducen el proceso como agentes que sirven de guía o modelos para el uso del lenguaje, para el razonamiento esperado por medio de la instrucción y para proveer las actividades y recursos que se pondrán en juego para propiciar el aprendizaje" (2009, p.5). Entonces, dado que la capacitación de Enciclomedia se centró en aspecto del manejo del software y de un posible proyecto pedagógico de las escuelas primarias -el cual es difícil creer que existiera-, no así un modelo educativo apoyado en las TIC, lo que se observó fue la praxis digital personal de los profesores hacia los alumnos que se basó en instrucciones de realización de actividades previamente establecidas y homogeneizadas. A su vez, habría que sumar que las edades, condiciones y prácticas digitales de los profesores son sumamente amplias por lo que la dinámica de cada aula quedó a merced de la propia comprensión de la narrativa de Enciclomedia. En su estudio de 2010 Martínez et al., señalan que se encontró que en los docentes de menos de 40 años hubo una mayor habilidad para usar la computadora y enciclomedia, mientras que a mayor edad disminuyó su uso y hubo menores logros con los alumnos, mismo grupo que sintió mayor disgusto 
al usar esta herramienta (2010) o básicamente "no son amigos de la computadora" (Loredo et al., 2010, p.13); de ahí que habría que pensar al docente de este proyecto como mediador-seleccionador (Leal y Arias, 2009) incluso sobre el propio software y que éste como uno de los agentes fueron parte del entramado de formación digital organizó el conocimiento, en esta mediación tecnológica, conforme a su propio relato digital y no sólo con criterios pedagógicos; los niveles de apropiación nunca fueron explorados de manera diagnóstica.

Es importante destacar que si bien el proyecto partió de la idea de integrar la tecnología en el aula de clases, ello no implicó una visión curricular, un modelo o proyecto educativo novedoso, ni provino de un planteamiento que hubiera hecho un diagnóstico y análisis previo para comprender o destacar el contexto nacional a nivel del uso de la tecnología educativa en el nivel básico de educación, las necesidades de uso o formación, o de las habilidades con las que ya contaban los de niños entre 10 y 12 años. En este sentido, no hubo una recuperación de la retórica digital de los involucrados, alumnos y profesorado, sino una del estado mexicano y a su vez de la idea generalizada de que la tecnología sumaba calidad, mejora y justicia social a la educación. La inserción de la tecnología educativa es diferente a la modificación de la práctica pedagógica, en el fondo no se trata de administrar los canales y medios digitales, sino de discutir las dinámicas y lo que soporta las prácticas en las aulas (Elizondo et al, 2006, p.221).

No habría que olvidar además, y esto es importante por el estudio presentado, que si bien en términos de la cobertura se alcanzaron en los primeros años equipamiento y capacitación en números aceptables considerando la complejidad del territorio nacional mexicano, en la práctica el proyecto no podía ser homogenizado en razón de las diferencias ya no sólo regionales, sino entre zonas urbanizadas (unas más centralizadas que otras como la Ciudad de México), rurales, indígenas, escuela tradicionales y multigrado, número de lenguas que se hablan, capacidades diferentes del alumnado, acceso a energía eléctrica y a conección a Internet; y en lo que respecta al tema que nos trata, porque las narrativas digitales entre profesores y alumnos no estaban empatadas en la misma dirección. 
Se trata de lo que Ertmer (1999), recuperado por Tinajero (2015), denomina barreras de primer orden que son obstáculos extrínsecos y de segundo orden que son dificultades personales, que se entrecruzan "debido a las complejidades que entrañan la integración de la tecnología en las escuelas" (Tinajero, 2015, p.348). Justo esta complejidad en el caso de los programas estatales de incorporación al ser evaluados, resaltan los aspectos principalmente de las barreras de primer orden, situándose principalmente en el aspecto de infraestructura así como de los contextos diferenciados de las aulas de clase (urbano-rural-indígena, gradomultigrado), pero tampoco hay un acercamiento sobre las barreras de segundo orden que podría también considerarse como subjetivas, en tanto que apelarían a la situación de los sujetos en distintos ámbitos y que considerando distintos entornos (transaccional, contextual, y microsistemas). La modificación de patrones de Enciclomedia al ser un ejercicio homogeneizado de digitalización socio-educativa, perdió de vista los comportamientos individuales y sociales de las diversas comunidades, "Ios lenguajes, los esquemas mentales y costumbres, lo cual demanda la sensibilización, la capacitación y el seguimiento, a fin de consolidar una cultura informática en las escuelas que propicie un uso crítico de esta tecnología" (Loredo et al., 2010, p.14).

Visto así, la retórica que se desprende de Enciclomedia fue la de una mediación tecnológica percibida sólo desde la interacción con los recursos, consignada desde el gobierno como proyecto más bien político modernizador. Esto también se percibe por los reportes que se generaron tanto por parte de la SEP como de otras entidades del gobierno mexicano como la Auditoría Superior de la Federación (ASF, 2010) y de otras instituciones que realizaron evaluaciones como de la Facultad Latinoamericana de Ciencias Sociales-México (FLACSO, 2018), cuyos reportes se centran principalmente en la relación del número de aulas equipadas, la respuesta de los proveedores de servicios para éstas, el número de profesores capacitados, asesores y capacitadores, la percepción de los beneficiarios del programa; no se observa en alguno de estos que se haya cruzado información entre las pruebas PISA, el rendimiento escolar, exámenes para el ingreso a secundaria, o a preparatoria, disminución de la deserción escolar, entre otros.

No obstante lo anterior, Enciclomedia tuvo un significado importante en la formación de habilidades y competencias digitales de los alumnos en 
tanto que significó su primer experiencia escolar en la que se integraron las TIC. Es por ello que este proyecto, más allá de su análisis en diversos niveles, debe ser considerado como parte de los entornos de formación digital. Enciclomedia se establece como parte de los diversos contextos culturales digitales que permiten reconstruir las prácticas. "Las construcciones generadas a partir de la colaboración entre los actores y mediadores, desarrolladas en escenarios y contextos diversos, impactaron diferenciadamente en las prácticas de cada uno de los actores" (Leal y Arias, 2009, p.7).

Con base en lo anterior el estudio propuesto buscó establecer una revisión de la percepción de universitarios sobre esta etapa de su formación digital en la primaria nueve años después, atendiendo simultáneamente al práctica y apropiación que actualmente tienen en la Universidad de las TIC. Esto en principio, nos debería permitir entender los aspectos que se han mantenido y cambiado, desde la praxis digital sobre la que se monta la cultura digital de estos jóvenes.

\section{Enfoque teórico-metodológico}

El estudio que se presenta parte de una visión compleja de la realidad, en este sentido se ha asumido que la prácticas digitales de los universitarios devienen de una formación rizomática en la que interactúan varios entornos con diversas experiencias. Para sostener que la retórica digital se forma de varias interacciones a lo largo de sus vidas académicas y subjetivas, el estudio tuvo un enfoque cualitativo.

En sentido el estudio recupera la posibilidad de discutir las trayectorias de formación digital de jóvenes universitarios mexicanos de manera no lineal. Lo anterior implica considerar que las rutas formativas de los participantes del estudio han tenido varios caminos y que su formación no se centra única y exclusivamente en la experiencia con Enciclomedia. Sin embargo, dado que el estudio se focaliza en la evaluación de esta política y su herramienta, casi 10 años después de que los jóvenes tuvieran esta experiencia, fue importante diferenciar el aspecto socio-digital de la formación académica digitalizada.

Para este estudio se contó con la participación de 39 estudiantes de la Facultad de Ciencias Políticas y Sociales, de la Universidad Nacional Autónoma de México. De éstos 29 son mujeres y 11 hombres; es 
importante señalar también que comprenden las edades de 19 a 24 años en su 5 ㅇ semestre. Más del $90 \%$ de los participantes provienen de la zona metropolitana (Ciudad de México y Estado de México), entre 2009 y 2010 se encontraban cursando el 50 y 60 grado de educación primaria. Por el contrario, fue fundamental reconocer que el resto de los estudiantes provienen de provincia y cursaron hasta el bachillerato en otros estados como Veracruz, Chiapas, Guerrero, Coahuila.

Este contexto es importante porque justamente la zona metropolitana de México fue el área que más se benefició del programa Enciclomedia, esto implicó no sólo acceso a los pizarrones, impresoras, computadoras, conexión a internet sino que los profesores recibieron capacitación por parte de la SEP.

Para el estudio se diseñaron y aplicaron dos instrumentos. El primero tiene que ver con las prácticas digitales que tienen los alumnos en términos de hábitos de consumo digital y, la relación con la tecnología educativa y sus procesos de aprendizaje; mientras que el segundo se centró en la recuperación de sus trayectorias formativas en el uso de tecnología no sólo desde el punto de vista de su experiencia con Enciclomedia, sino sus primeros contactos con la tecnología por cuenta propia. El cuadro 2 muestra la composición de los instrumentos.

Cuadro 2. Composición de los instrumentos aplicados.

Instrumento 1

\begin{tabular}{clcl}
\hline Hábitos digitales & 7 reactivos & $\begin{array}{c}\text { Experiencia con } \\
\text { Enciclomedia }\end{array}$ & 5 reactivos \\
\hline $\begin{array}{c}\text { Prácticas educativas } \\
\text { digitales }\end{array}$ & 13 reactivos & $\begin{array}{c}\text { Experiencias } \\
\text { escolares } \\
\text { posteriores con las } \\
\text { TIC }\end{array}$ & 2 reactivos \\
\hline $\begin{array}{c}\text { Relación con } \\
\text { dispositivos celulares }\end{array}$ & $\begin{array}{c}3 \text { preguntas } \\
\text { abiertas }\end{array}$ & $\begin{array}{c}\text { Experimentación } \\
\text { con tecnología } \\
\text { digital }\end{array}$ & 2 reactivos \\
\hline
\end{tabular}

Fuente: Elaboración propia

Instrumento 2 
Como se ha señalado, el enfoque del estudio buscó mostrar cómo las trayectorias formativas hacia las competencias digitales no siguen un camino lineal, sino que son rizomáticas. En este sentido, si bien se discutirá el impacto después de 19 años de las estrategias con el uso de Enciclomedia, es importante recuperar los propios procesos de los estudiantes en su formación en otros niveles y entornos. La figura 2 muestra la propuesta del estudio. En ella se puede observar que los rizomas nos explican que la trayectoria y la formación digital no es lineal, sino que procede de distintas situaciones. Para el estudio se consideró la experiencia con Enciclomedia pero también la experimentación con la tecnología digital, el uso social de tecnologías digitales y la paulatina maduración para la integración de las TIC en el trabajo universitario. Todos estos aspectos generan distintos entornos sobre los que cada estudiante se va formando y adquiriendo distintas habilidades y que posteriormente se materializan como competencias digitales. Partiendo de esta visión compleja rizomática se analizaron los resultados del estudio.

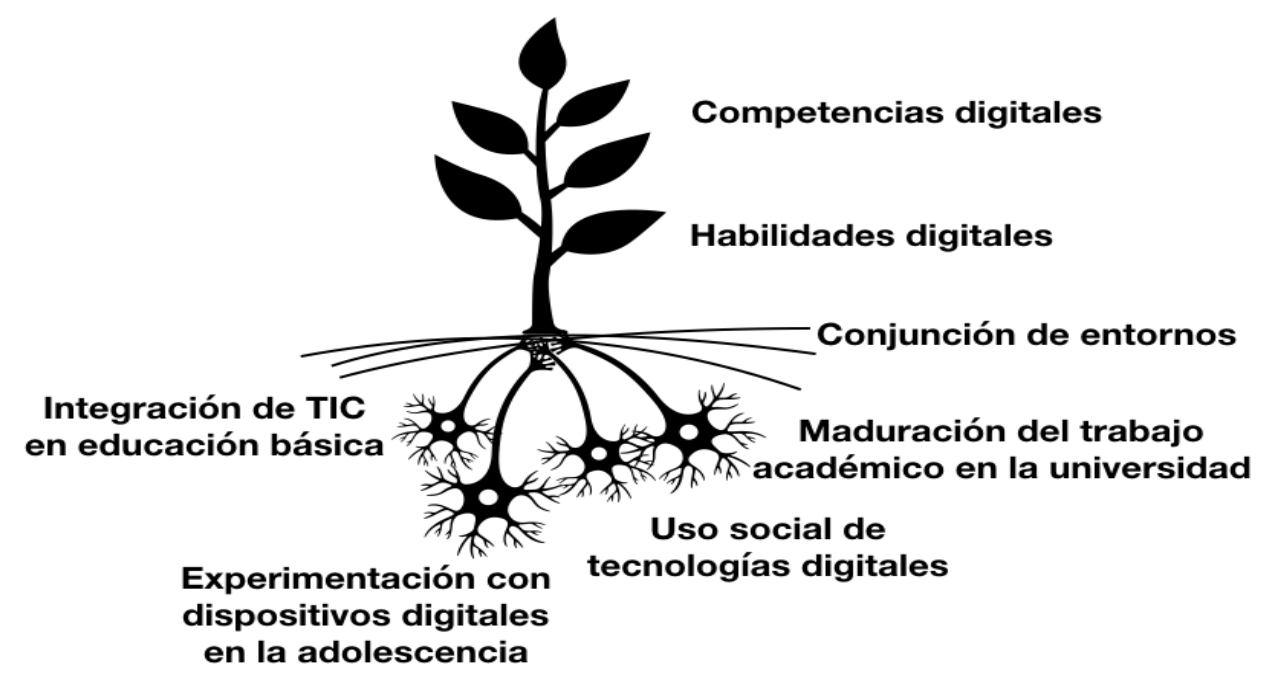

Figura 2. Visión rizomática y compleja del estudio Fuente: Autoría propia

Los cuatro aspectos señalados bajo tierra de la figura 2, son los que se analizaron mediante los datos que se obtuvieron de estudio aplicado y que a continuación se presentan.

\section{Resultados}


Como se mencionó los universitarios participantes del estudio señalaron principalmente los años 2010 y 2011 como aquellos en los que cursaron 5 o o 6 o de primaria, aunque sobresale este período, los datos mostraron a una parte de los alumnos que cursaron estos grados entre 2004 y 2006. Lo anterior es importante señalarlo porque la primera etapa de Enciclomedia se dio justo en estos años mientras que la mayoría de los estudiantes del estudio habrían estado en la última parte del proyecto. En este sentido se habría esperado que para este segundo período se hubieran subsanado algunos aspectos principalmente de uso de la herramienta. La gráfica 1 muestra los años que refieren haber estado en los últimos grados de primaria, en amarillo aparecen las mujeres y en azul los hombres.

Gráfica 1. Años en los que los estudiantes universitarios señalan haber cursado el 5ㅇ o 6으 grado de primaria.

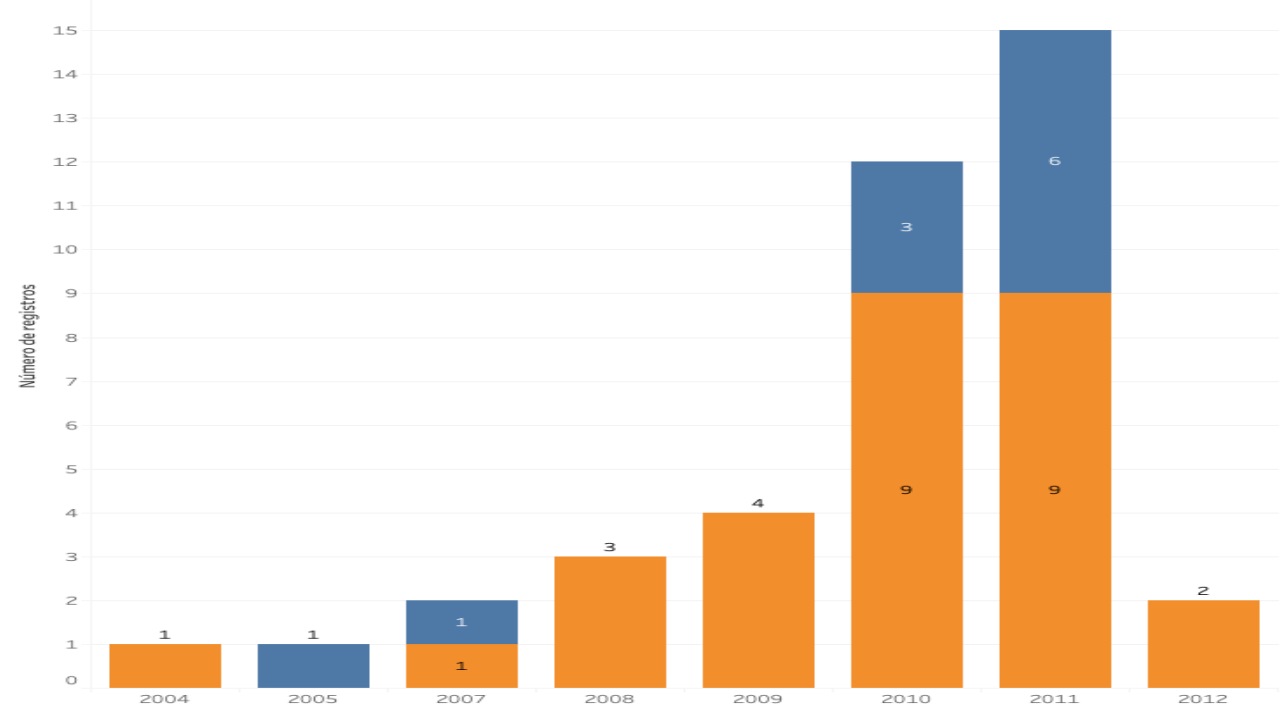

No obstante lo anterior, cuando se les preguntó quienes recuerdan haber usado Enciclomedia, la percepción cambió porque si bien la mayoría señaló usarla (28), hubo algunos en que no recordaron sí lo habían empleado (8) y otros que dijeron no haberla empleado (3). Pero en el caso de quienes sí lo hicieron, la percepción se diferencia notablemente entre mujeres y hombres, puesto que mientras la mayor parte de éstos últimos tienen la idea de que no les benefició para su aprendizaje, en el caso de las mujeres la cantidad de quienes observaron una mejora de quienes no, es prácticamente la misma. La gráfica 2 muestra esta situación, en rojo aparecen la percepción positiva y en azul la negativa. 
Gráfica 2. Uso de Enciclomedia en la primaria y percepción de beneficio.

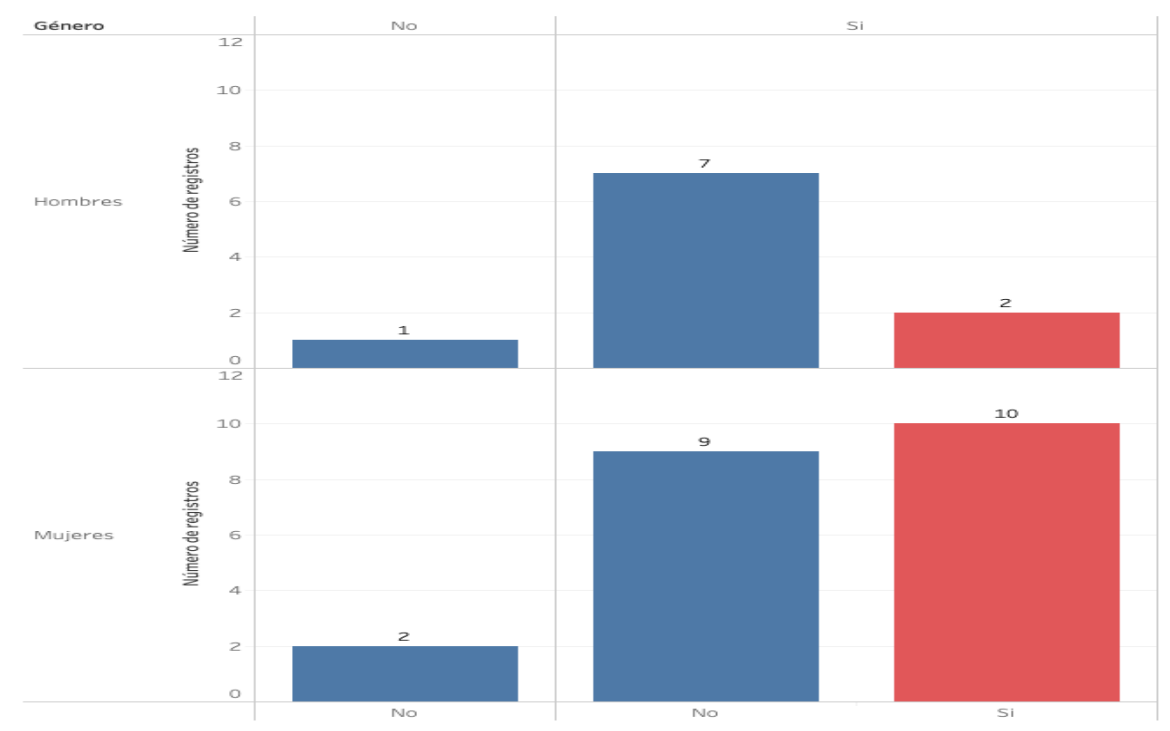

En cuanto a los materiales que recuerdan haber empleado los universitario en su niñez de Enciclomedia, la figura 3 muestra las relaciones de recursos y sobresale el uso sencillo de el pizarrón, los mapas y los libros digitalizados, lo que coincide con algunos de los estudios que señalaron que su mayor uso se dio para las clases de español, historia y geografía. Muy poco uso de la galería de arte, la filmoteca, visitas guiadas, la Red Escolar o SEPiensa.

Figura 3. Priincipales recursos usados de Enciclonedia reconocidos por los universitarios 


\section{prociênci@s}

\section{v. 2, n. 2, dezembro, 2019}

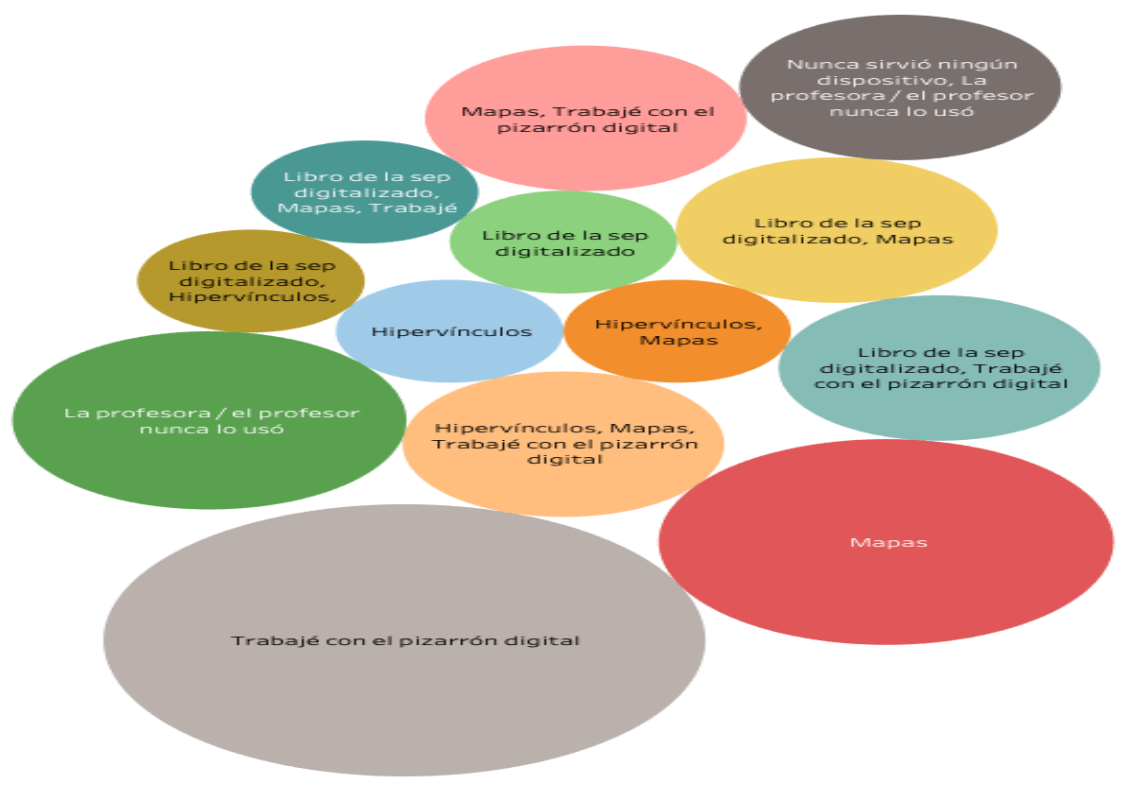

Por otro lado, la percepción sobre sí consideran que aprovecharon la experiencia con Enciclomedia en la primaria, no revela un aspecto positivo mayoritario, sólo algunas mujeres manifestaron que fue regular (gráfica 3).

Gráfica 3. Percepción de aprovechamiento de los jóvenes cuando usaron en su niñez Enciclomedia

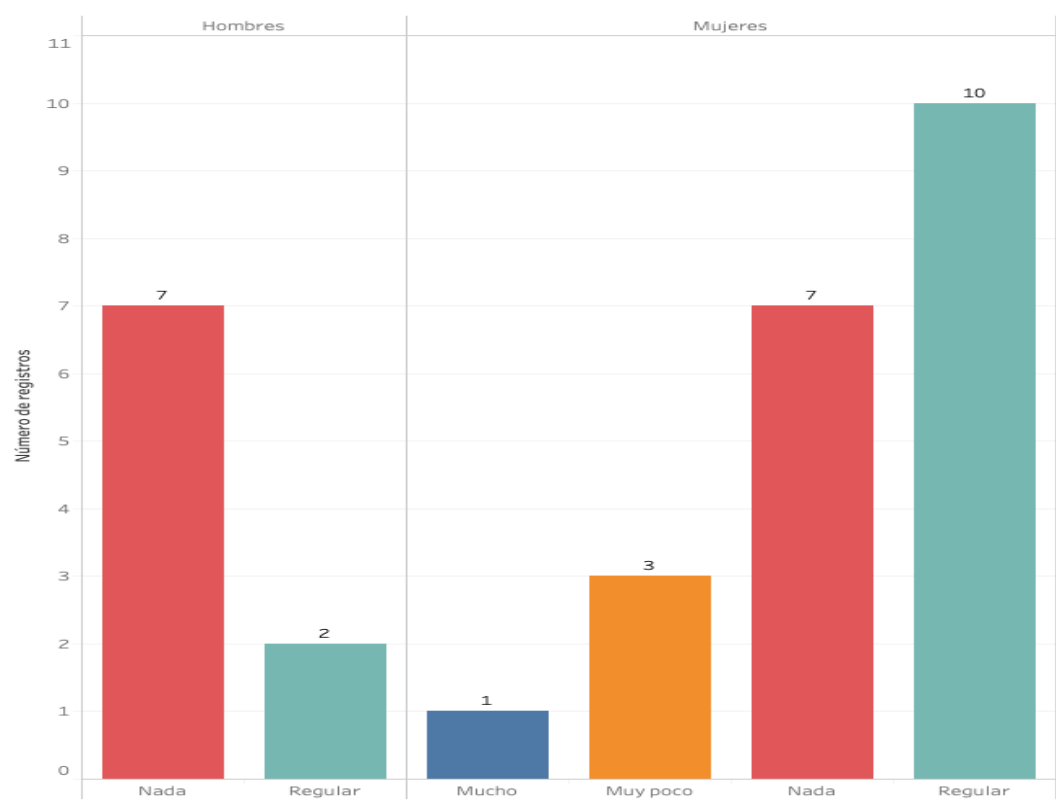

A la pregunta de con qué tipo de formación digital se identificaban más (gráfica 4) buena parte de los jóvenes de ambos sexos (9-12) dijeron haber aprendido de manera autónoma utilizando ellos mismos dispositivos 
y herramientas (color verde), pero una parte importante de las mujeres (12) consideran los cursos escolares previos a la universidad como parte de su formación digital (color rojo); mientras que sólo hubo un caso masculino que reportó haberse apoyado en sus amigos (color amarillo) y cinco femeninos que alguien externo de la escuela le enseñó (color azul).

\section{Gráfica 4. Autodentificación con el tipo de formación digital}
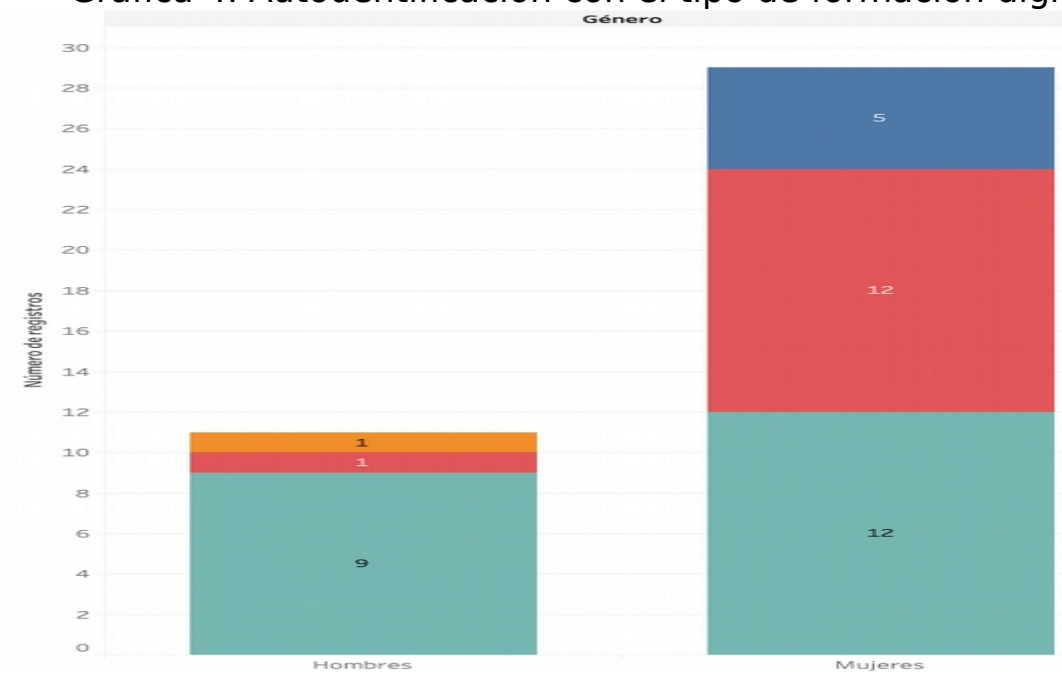

Finalmente, resultó llamativo observar que a la pregunta de cuál fue la primera interacción digital que recuerdan, los participantes la asociaron principalmente con el celular de sus padres, uso de diversos $C D$, videojuegos y ver videos. En buena medida, esto fortalece el argumento de que se trata de personas que crecieron inmersos en la experiencia visual y auditiva pese a que no reconozcan la experiencia de Enciclomedia.

Gráfica 5. Principales relaciones de primeras interacciones digitales recordadas por los jóvenes universitarios. 


\section{prociênci@s}

\section{v. 2, n. 2, dezembro, 2019}

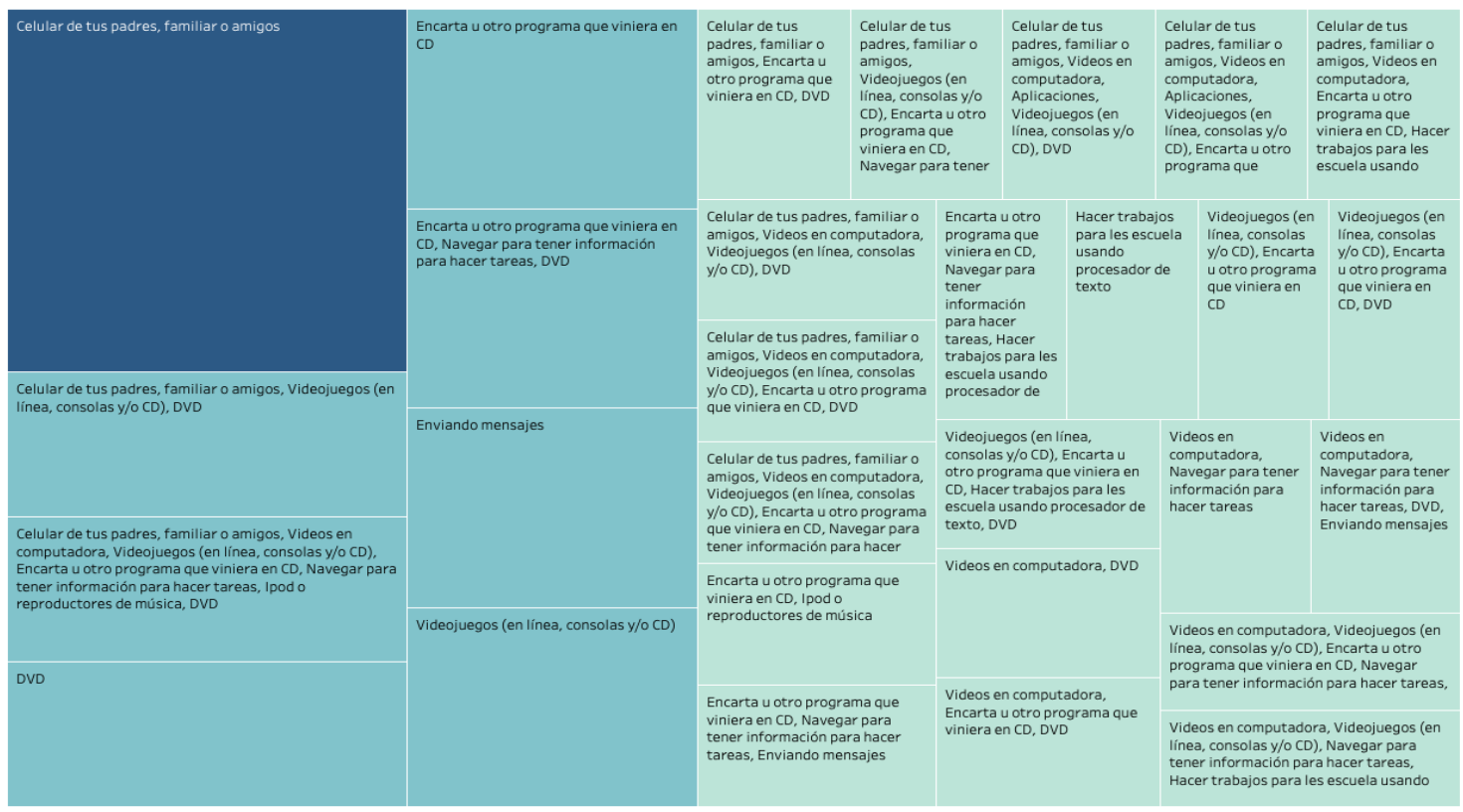

Este entramado de primeras prácticas digitales nos muestran una clara evolución cuando se analizan los niveles de consumo de los participantes, en una dinámica de apropiación de la tecnología digital en aspectos como la navegación, mensajería, el uso de redes sociales o de streaming, videos y la lectura en dispositivos (gráfica 6). Aunque los contenidos audiovisuales son una variable permanente, llama la atención que de estas actividades sea la lectura en dispositivos móviles y la navegación (lo que implica la dinámica del hipertexto) las actividades que reportaron niveles muy altos de consumo. Y que la autopercepción en otras funciones sea principalmente de un consumo medio.

Gráfica 6. Autopercepción de niveles de consumo en diversas actividades digitales.
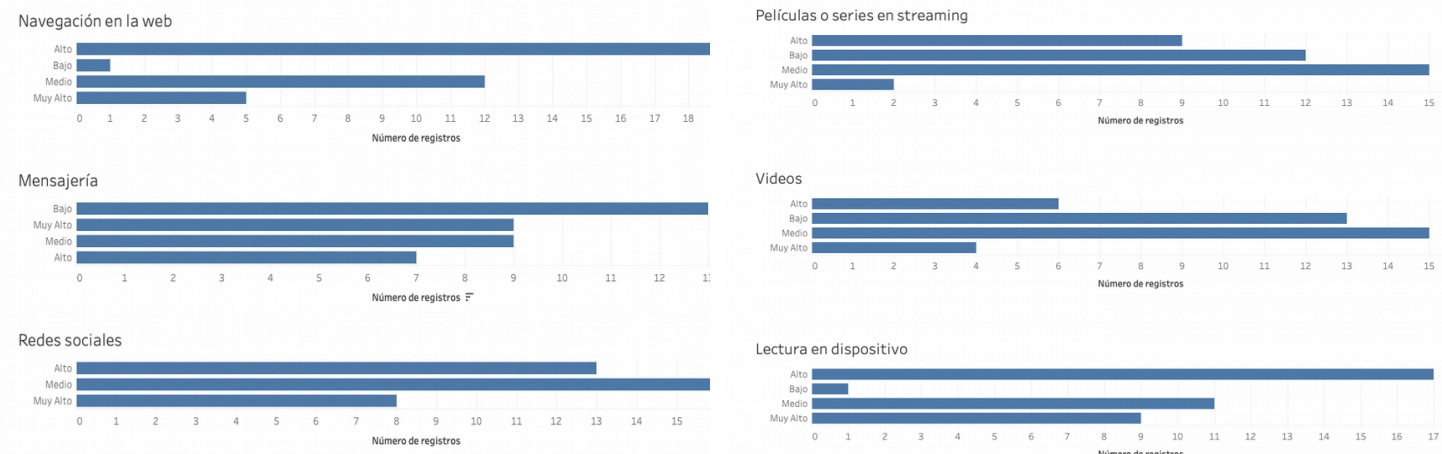

En este sentido, y partiendo de que la integración de las TIC en la vida académica de se ha visto desde el uso de recursos y no desde la 
óptica de la cultura digital así como del factor cognitivo, la gráfica 7 muestra que la apreciación de los universitarios participantes del estudio, consideran que un audio, un video o una infografía, materiales principalmente audiovisuales, si bien tienen una importancia (11) o son moderadamente importantes (13), frente a una lectura, no son del todo determinantes como material de estudio. En este sentido se puede establecer un criterio para separar materiales de consulta o de apoyo, de aquellos que tienen un valor fundamental para la formación universitaria.

Gráfica 7. Importancia de videos, audios o fotografías frente a una lectura como parte de los materiales de estudio.

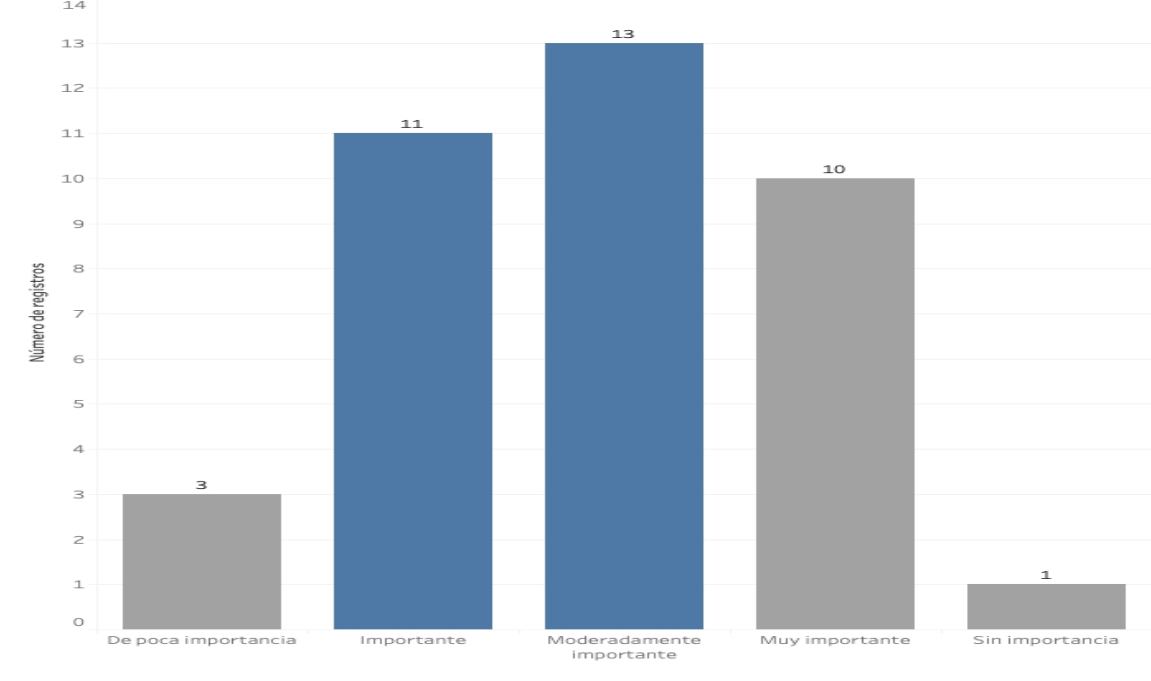

Por otra parte, en este mismo ejercicio de rescatar la retórica de la práctica digital de los universitarios en relación a su formación, se les realizó una pregunta abierta sobre los aspectos positivos y negativos de la relación con su dispositivo móvil (figura 4). Esta información se procesó en el programa MaxQDA para formar una red semántica tomando como punto de referencia la propia pregunta. Se encontró que la mayor parte de los alumnos del estudio encontraron aspectos positivos a la relación (34) y en principio la visualizan como una herramienta (31), si bien en otros casos vieron también los aspectos negativos principalmente (29) siendo el más fuerte la distracción (15) y la dependencia (14). Es importante señalar que hubo un caso que no contestó.

Figura 4. Red semántica de aspectos positivos y negativos de la relación con los dispositivos móviles. 


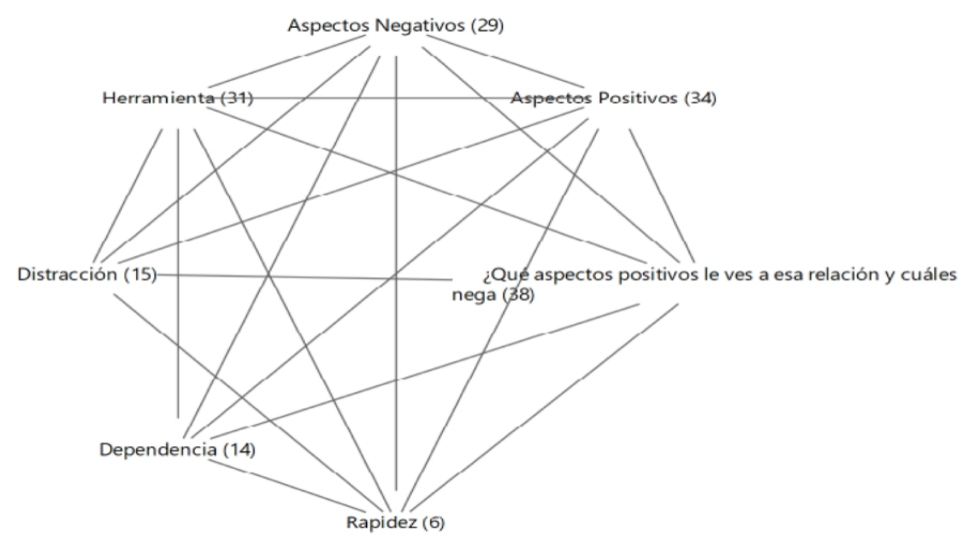

Finalmente, se les preguntó también de manera abierta cómo les gustaría que se usara la tecnología digital para fortalecer sus estudios universitarios. La figura 5 muestra dos condiciones que coinciden con la retórica audiovisual que los jóvenes tienen desde que se empezaron a formar en el uso de estas herramientas. El principal recurso que apareció en las respuestas fue el empleo de materiales audiovisuales (20) pero en segundo lugar la de lecturas y literatura digitales (11) que coinciden con la importancia que le asignan a esta actividad, ya antes referido, pero desde los dispositivos digitales.

Figura 5. Red semántica sobre los recursos digitales que deberían integrar los docentes universitarios en sus materias

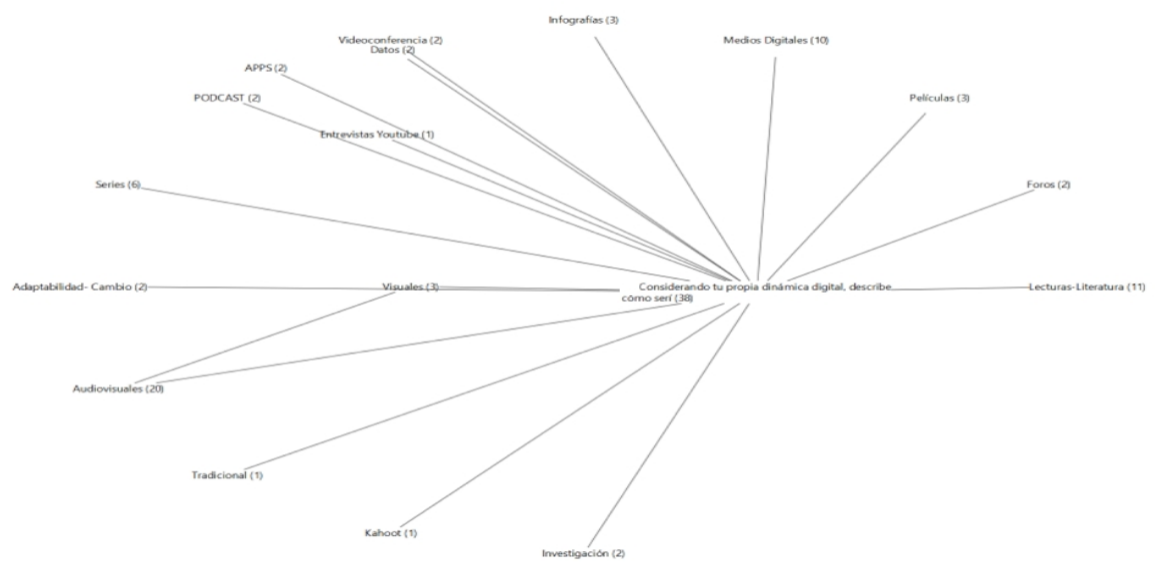

\section{Discusión y conclusión}


La contrastación de las retóricas entre los proyectos educativos de los gobiernos frente a la de los beneficiados, nos permiten obtener una lectura diferente de la evolución de los agentes, en este caso de los estudiantes universitarios. Si bien es cierto que la imposición del modelo de Enciclomedia chocó con los profesores principalmente por las diferenciadas retóricas de formación y de práctica digital, en el caso de los niños en ese momento, formó parte de su acercamiento desde el aula de las TIC. En este sentido la integración éstas en la educación básica, más allá de la valoración positiva o negativa, es parte de la historia de los hoy universitarios. Puesto que la mayor parte de sus experiencias digitales se dieron fuera del entorno educativo, en el familiar y por experimentación propia, aunque haya un cierto reconocimiento de la importancia del programa como experiencia inicial y se reconoce la formación que se puede dar antes del ingreso a los estudios universitarios.

Así pues, de manera rizomática el uso escolar, el acercamiento familiar pero sobre todo la apropiación social de la cultura digital, es lo que permite entender que los distintos entornos en los que se desarrollaron los universitarios, dan como resultado una formación no lineal sino rizomática, no sólo en términos de la adquisición de habilidades y competencias, un sentido cognitivo del mundo digital. Lograr diferenciar un material de apoyo, de uno que constituye la base de un curso, pese a tener una dinámica audiovisual de aprendizaje desde la primaria, nos habla de maduración del trabajo universitario. Los videos y recursos auditivos y visuales, acompañan el aprendizaje, pero no sustituyen el proceso que se lleva a cabo con la lectura. En otras palabras, se pudo observar que ese mismo esquema rizomático permanece y se adapta en diversas circunstancias.

Si bien no se trata de una norma, es importante destacar el entorno familiar en el que se desarrollaron los alumnos del estudio, si bien en muchos de ellos destacan su autoformación en el uso de tecnología, han vivido en ambientes con conexión a Internet desde muy pequeños. Además, considerando que los jóvenes que logran a ingresar a la UNAM son pocos en relación a la cantidad de solicitudes para el examen de admisión tanto para su nivel de bachillerato como de estudios universitarios, son personas que provienen de áreas urbanizadas y la mayoría de los casos con alta densidad poblacional, lo que implica mayor necesidad de servicios e interconexión; pero también destaca el hecho de 
que en sus escuelas si se haya usado Enciclomedia y que si bien la percepción no es del todo favorable, coincide el entorno que destacan Martínez et al. (2010) respecto a que los mayores logros se encontraron en escuelas urbanas cuyos directores mostraron preocupación por dar cumplimiento a los programas, constantemente reportando el avance, con mayor índice de estudios formativos, mejor comunicación con los padres de familia, con sedes más limpias y en mejores condiciones y que de manera particular valoraban la importancia del acceso a Internet porque ellos mismos contaban en sus hogares con conexión (Martínez et al. 2010, p.26-27). Este aspecto nos importa porque se puede entender que la cantidad de enlaces rizomáticos que coadyuvaron en la cultura y retórica de los jóvenes universitarios proviene de distintos agentes. En este sentido hay un entramado que efectivamente dice que tanto la cultura como la retórica que se ha construído de manera personal sobre la tecnología ha pasado por varios momentos, y que el hecho de que hayan desarrollado habilidades y se encuentren en ese paso intermedio hacia las competencias digitales profesionales, se viene dando desde hace por lo menos 10 años.

No obstante lo anterior, habría que establecer que la mayoría de los niños que cursaron primaria en estos años, además de estar en otros entornos digitales en los que estuvieron inmersos, la actividad que tuvieron con Enciclomedia se situó como la primera experiencia que tuvieron en el ámbito de su formación académica. Es por eso que más allá del análisis de las fortalezas y debilidades del propio programa, también es importante verlo en el contexto de la formación de una cultura digital de los hoy universitarios, que en conjunto con los otros contextos dan una praxis digital personalizada. Es cierto que se pudieron mejorar otros aspectos como el pensamiento matemático y computacional, o la mejora de la comprensión lectora, pero estos no fue planteado como tal en el proyecto original, no obstante, de la retórica digital de los gobiernos bajo los que se dio Enciclomedia, la de los docentes, los familiares y amigos, la de la web de inicios del siglo $X X$, generaron un entramado rizomático que apuntaron a la formación paulatina de habilidades y a posteriori de competencias digitales, que se visualizan hoy en día en el ámbito universitario.

En consecuencia, la retórica digital de los universitarios, considerando su origen formativo, y dando seguimiento a sus prácticas 
sociales y académicas, es rizomática y es altamente probable que se mantenga así. Sin embargo, el sentido y maduración que le dan como universitarios a la narrativa digital, les permite del mismo modo no lineal valorar los recursos entre formativo, de apoyo y recreativos. Con esto se puede entender que cada estudiante va formando su propia dinámica a partir de su práctica, por lo que la integración de las TIC a edad temprana en buena medida fortaleció esta práctica considerando que se trató del primer programa masivo de este tipo, si bien los estudios muestran que no se alcanzaron los objetivos pedagógicos.

\section{Bibliografía}

AUDITORÍA SUPERIOR DE LA FEDERACIÓN. Informe del Resultado de la Fiscalización Superior de la Cuenta Pública 2010, Programa Enciclomedia (SEP). México: Grupo Funcional Desarrollo Social, 2010. Disponible en: <https://www.asf.gob.mx/Trans/Informes/IR2010i/Indice/Auditorias.htm> . Acceso: septiembre, 2019.

BOLTER, J. D. Writing space: Computers, hypertext, and the remediation of print. New York: Routledge, 2001.

ERTMER, P. Addressing first -and second- order barriers to change: Strategies for technology integration. Educational Technology Research and Development, 1999.

ELIZONDO HUERTA, A., PAREDES OCHOA, F. J., Y PRIETO HERNÁNDEZ, A. $M$. Enciclomedia. Un programa a debate. Revista Mexicana de Investigación Educativa, 11(28), 209-224, 2006. Disponible en:

$<$ https://www.redalyc.org/pdf/140/14002811.pdf> Acceso: septiembre, 2019.

EYMAN, D. Digital rhetoric: Theory, method, practice. University of Michigan Press: 2015. Disponible en: <https://doi.org/10.1111/14678284.00096> Acceso: septiembre, 2019.

FACULTAD LATINOAMERICANA DE CIENCIAS SOCIALES-MÉXICO. Informe programa Enciclomedia. México: 2018. Disponible en:

$<$ https://www.sep.gob.mx/es/sep1/programa_enciclomedia> Acceso:

septiembre, 2019.

HESS, A. (2017). Introduction. Theorizing digital rhetoric. New York: Routledge, 2017. Disponible en: 
<https://www.taylorfrancis.com/books/e/9781351788649/chapters/10.432 4\%2F9781315203645-7> Acceso: septiembre, 2019.

LANDOW, G. Hyper/Text/Theory. Johns Hopkins University Press, 1994.

LANHAM, R. A. Digital rhetoric: Theory, practice, and property en Literacy online: The promise (and peril) of reading and writing with computers, 221-243. University of Pittsburgh Press, 1992.

LANHAM, R. A. The electronic word: Democracy, technology, and the arts. University of Chicago Press, 1993, 2010 (reimpresión).

LÉVY, P. Cibercultura: informe al Consejo de Europa (No. 16). Anthropos Editorial, 2007.

LOSH, E. M. Virtualpolitik: An electronic history of government media-making in a time of war, scandal, disaster, miscommunication, and mistakes. MIT Press, 2009. Disponible en: $<$ https://books.google.es/books?

id =hC35pomwgrgC\&lpg=PR7\&ots =jaF0P6xz4D\&dq=losh $\% 20$ elizabeth\&lr\&hl=es\&pg=PR7\#v=onepage\&q=losh \%20elizabeth\&f=false $>$ Acceso: septiembre, 2019.

LEAL ESPINOZA, M.; ARIAS LÓPEZ, J. L. Enciclomedia: diagnóstico y propuesta de mejora desde las necesidades de los actores. Ponencia presentada en el X Congreso Nacional de Investigación Educativa: México, 2009. Disponible en:

<http://www.comie.org.mx/congreso/memoriaelectronica/v10/contenido/ contenido0107T.htm> Acceso: septiembre, 2019.

LOREDO ENRÍQUEZ, J.; GARCÍA CABRERO, B.; ALVARADO GARCÍA, F. Identificación de necesidades de formación docente en el uso pedagógico de Enciclomedia en Sinéctica, n. 34), 1-16, 2010. Disponible en: <http://www.scielo.org.mx/scielo.php?pid=S1665109X2010000100003\&script=sci_arttext\&tIng=en> Acceso: septiembre, 2019.

MARTÍNEZ RODRÍGUEZ, F.; ORTEGA CANTO, M.; QUESADA GARCÍA, J.; SÁNCHEZ ZÚÑIGA, M. (2010). Evaluación de Enciclomedia en Revista Latinoamericana de Estudios Educativos, n.40, v.2, 9-36. Disponible en: $<$ https://www.redalyc.org/pdf/270/27018884002.pdf> Acceso: septiembre, 2019.

MOULTHROP, S. Rhizome and resistance: Hypertext and the dreams of a new culture en Hyper/text/theory, 1994, p.299-319. 
PFISTER, D. S. (2014). Networked media, networked rhetorics: Attention and deliberation en The early blogosphere, v. 10, Penn State Press. Disponible en: <https://muse.jhu.edu/book/47358> Acceso: septiembre, 2019.

\section{SECRETARÍA DE EDUCACIÓN PÚBLICA. Libro Blanco Programa}

Enciclomedia 2006-2012: México, 2007. Disponible en: $<$ https://www.sep.gob.mx/work/models/sep1/Resource/2959/4/images/LB \%20Enciclomedia.pdf> Acceso: septiembre, 2019.

SUBSECRETARÍA DE EDUCACIÓN BÁSICA Y NORMAL. Secretaría de Educación Pública. Programa Enciclomedia, documento base: México, 2004. Disponible en:

< https://www.oei.es > mexico > documento enciclomedia > Acceso: septiembre, 2019.

ZALLO, R. Civilización y vida social. Paradojas de la cultura digital en TELOS 88: La Cultura Digital, 88, 103, 2011. Disponible en:

$<$ https://www.fundaciontelefonica.com/arte cultura/publicaciones-listado/ pagina-item-publicaciones/itempubli/256/> Acceso: septiembre, 2019.

ZAPPEN, J. P. (2005). Digital rhetoric: Toward an integrated theory en Technical Communication Quarterly, n.14, n.3, 2005, 319-325.

Disponible en: <https://doi.org/10.1207/s15427625tcq1403_10>Acceso: septiembre, 2019. 